\title{
Efficacy of respiratory tele-rehabilitation in COPD patients: Systematic review and meta-analysis
}

\author{
Josuel Ora ${ }^{1}$, Emanuela Prendi ${ }^{2}$, Maria Laura Attinà ${ }^{2}$, Mario Cazzola $^{3}$, Luigino Calzetta ${ }^{4}$, \\ Paola Rogliani ${ }^{1,3}$ \\ ${ }^{1}$ Division of Respiratory Medicine, University Hospital Policlinico Tor Vergata, Rome, Italy; ${ }^{2}$ Department of \\ Biomedical Sciences, Catholic University "Nostra Signora del Buon Consiglio", Tirana, Albania; ${ }^{3}$ Unit of Respiratory \\ Medicine, Department of Experimental Medicine, University of Rome "Tor Vergata", Rome, Italy; ${ }^{4}$ Department of \\ Medicine and Surgery, Respiratory Disease and Lung Function Unit, University of Parma, Italy
}

\begin{abstract}
Pulmonary rehabilitation (PR) is a proven and effective intervention for chronic obstructive pulmonary disease (COPD). The recent pandemic has raised interest on new services, such as telerehabilitation (Tele-R). The aim of this study was to systematically
\end{abstract}

Correspondence: Dr. Josuel Ora, Division of Respiratory Medicine, University Hospital "Policlinico Tor Vergata", Viale Oxford 81, 00133 Rome, Italy.

Tel.+39.347.6177430. E-mail: josuel.ora@ptvonline.it

Key words: Tele-rehabilitation; COPD; exercise tolerance; 6MWT; mMRC; CAT.

Conflicts of interest: All authors declare no conflicts of interest related to this paper.

Contributions: JO, EP, MLA, MC, LC, PR, had full access to all the data in the study and take the responsibility for the integrity of the data and the accuracy of the data analysis; JO, EP, MLA, designed the statistical analyses, original manuscript drafting; MC, LC, PR, consultation on statistical analyses and data interpretation; JO, EP, guarantors of this review and meta-analysis. All authors revised the article critically for important intellectual content, gave final approval of the version to be published, and agreed to be accountable for all aspects of the article in ensuring that questions related to the accuracy or integrity of any part of the article were appropriately investigated and resolved.

Received for publication: 27 September 2021.

Accepted for publication: 23 December 2021.

Publisher's note: All claims expressed in this article are solely those of the authors and do not necessarily represent those of their affiliated organizations, or those of the publisher, the editors and the reviewers. Any product that may be evaluated in this article or claim that may be made by its manufacturer is not guaranteed or endorsed by the publisher.

${ }^{\circ}$ Copyright: the Author(s), 2022

Licensee PAGEPress, Italy

Monaldi Archives for Chest Disease 2022; 92:2105

doi: 10.4081/monaldi.2022.2105

This article is distributed under the terms of the Creative Commons Attribution-NonCommercial International License (CC BY-NC 4.0) which permits any noncommercial use, distribution, and reproduction in any medium, provided the original author(s) and source are credited. review the effects of Tele-R in COPD on: i) exercise capacity evaluated by the 6-minute walk test (6MWT); ii) dyspnea (modified Medical Research Council - mMRC); 3) COPD control (the COPD assessment test - CAT). The analysis compared Tele-R versus no rehabilitation and Tele- $\mathrm{R}$ versus center-based rehabilitation. This meta-analysis was undertaken according to PRISMA recommendations. This pair-wise meta-analysis included data obtained from studies that enrolled 758 COPD patients. The tele-R compared to no rehabilitation improved the 6MWT distance of $48 \mathrm{~m}$ (CI: 24, 72; $\mathrm{p}<0.001)$ and the mMRC of $-1.02 \mathrm{U}(\mathrm{CI}:-1.49,-0.59 ; \mathrm{p}<0.001)$, and the CAT of $-5.74 \mathrm{U}$ (CI: $-7.42,-0.407 ; \mathrm{p}<0.001)$. The tele-R compared to center-based rehabilitation showed no difference on 6MWT distance $(\mathrm{p}=0.563), \mathrm{mMRC}(\mathrm{p}=0.911)$, and CAT $(\mathrm{p}=0.85)$. In COPD patients, Tele- $\mathrm{R}$ is effective in improving exercise tolerance and patient-reported outcomes and it seems to be a valid alternative to center-based rehabilitation, but more studies are needed to better understand how to select the right patients and which kind of Tele- $\mathrm{R}$ is more appropriate.

\section{Introduction}

Pulmonary rehabilitation (PR) is a proven and effective intervention for people with chronic respiratory diseases including chronic obstructive pulmonary disease (COPD) [1]. In COPD patients, PR improves symptoms, disease control, and emotional state [2], although an improvement in activity levels in daily life is not always demonstrable [3].

PR is traditionally delivered by a physiotherapist in a residential hospital or outpatient clinic. However, due to factors related both to the scarcity of territorial rehabilitation facilities and rehabilitation programs and to the logistical or health difficulties of patients, it is very often reserved for the most serious patients only therefore not everyone can access it [4]. In addition, the recent SARS-CoV-2 epidemic has further reduced the number of available rehabilitation facilities, dedicating some of them to COVID-19 patients or reducing the number of beds for the risk of contagion or closing centers. On the other hand, the same epidemic experience has shown how some alternative models of service delivery such as tele-medicine and tele-rehabilitation (Tele-R) can assist and replace medicine in general and rehabilitation in particular [5].

Tele- $\mathrm{R}$ is the provision of remote rehabilitation services, using information and communication technologies. There are several models of Tele-R and they can be supplemented with face-to-face rehabilitation [6]. The purpose of all Tele- $\mathrm{R}$ is to bring the service 
to the patient and not the patient to the service, clinic or hospital.

This meta-analysis aims to evaluate the clinical efficacy of Tele- $\mathrm{R}$ in COPD patients and specifically its effectiveness in improving exercise tolerance and patient reported outcomes (PRO). Since Tele-R can be compared both with a group not undergoing rehabilitation (control group - CTRL) and with a group undergoing traditional rehabilitation (center-based rehabilitation), the objective of this meta-analysis is to demonstrate the superiority of Tele-R over the CTRL group and the non-inferiority compared to traditional rehabilitation.

\section{Methods}

\section{Research strategy and eligibility of studies}

This pairwise analysis was performed in accordance with Preferred Reporting Items for Systematic Review and MetaAnalysis Protocols (PRISMA-P)[7], as shown in the diagram flow shown in Figure 1. This study met all the recommended items reported by the PRISMA-P checklist [8] and submitted to PROSPERO (ID: 276848).

The PICO (Patient problem, Intervention, Comparison, and Outcome) scheme was applied to develop the research strategy and demand of the literature, as previously reported. That is, the "Patient problem" included only patients with COPD; “ Intervention" considered rehabilitation in telemedicine (respiratory Tele-R); " Comparison" included patients receiving Tele-R compared to those receiving center-based rehabilitation or no rehabilitation; the "Outcomes" included both exercise tolerance and PRO, specifically dyspnea measured as mMRC and CAT. A comprehensive literature search of randomized controlled trials (RCTs) was performed to consider respiratory Tele- $\mathrm{R}$ in patients with respiratory diseases. Prospective or retrospective non-randomized studies were discarded.

The search was performed in MEDLINE and Google Scholar, in order to predict the relevant studies published until August $30^{\text {th }} 2021$.

The searched words were [telerehabilitation OR tele-rehabilitation] AND pulmonary rehabilitation" and the search string was as follows: ("telerehabilitation"[MeSH Terms] OR "telerehabilitation"[All Fields] OR ("telerehabilitation"[MeSH Terms] OR "telerehabilitation"[All Fields] OR ("tele"[All Fields] AND "rehabilitation"[All Fields]) OR “tele rehabilitation"[All Fields])) AND (("lung"[MeSH Terms] OR "lung"[All Fields] OR "pulmonary"[All Fields]) AND ("rehabilitant”[All Fields] OR "rehabilitants"[All Fields] OR "rehabilitate"[All Fields] OR "rehabilitated"[All Fields] OR "rehabilitates"[All Fields] OR "rehabilitating”[All Fields] OR "rehabilitation"[MeSH Terms] OR "rehabilitation"[All Fields] OR "rehabilitations"[All Fields] OR "rehabilitative"[All Fields] OR "rehabilitation"[MeSH Subheading] OR "rehabilitation s"[All Fields] OR "rehabilitational"[All Fields] OR "rehabilitator"[All Fields] OR "rehabilitators"[All Fields])).

Subsequently, the research was limited only to COPD by eliminating the articles that referred to other pathologies.

In addition, previously published meta-analysis citations and literature reviews on the subject were verified to select additional relevant studies $[9,10]$.

\section{Selection of studies}

All RCTs concerning Tele-R in patients with COPD were selected in accordance with previously mentioned criteria and included in the quantitative analysis. Two reviewers independently audited the studies reviewed and any differences of opinion on the selection of studies were resolved by consensus.

\section{Data extraction}

Data from the included studies were extracted and controlled for study characteristics, number and characteristics of patients analyzed, age, sex, forced expiratory volume in 1 second $\left(\mathrm{FEV}_{1}\right)$, 6MWT and PRO. Data were extracted in accordance with recommendations of Data Extraction for Complex Meta-anALysis (DECiMAL) [11].

\section{Endpoint}

The main endpoints of this pairs meta-analysis were the impact of Tele-R in patients with COPD on exercise tolerance, measured as the distance covered in meters during 6MWT and on PRO evaluated through questionnaires (CAT, mMRC).

\section{Risk of bias}

The heterogeneity test $\left(\mathrm{I}^{2}\right)$ was performed to quantify the dissimilarity between studies, as previously reported [12].

\section{Data summary and analysis}

A pairs meta-analysis was performed to determine the impact of Tele-R in patients with COPD. The results were expressed as mean difference (MD) and 95\% confidence interval (95\% CI).

Since the data were selected from a number of studies performed by researchers operating independently and it is not possible to assume a common effect size, a binary model of random effects was used to balance the weights of the studies and adequately estimate $95 \% \mathrm{CI}$ of the average distribution of the effect of PR on the variables studied [13].

OpenMetaAnalyst software [12] was used to perform metaanalysis in pairs and to make data graphs. Statistical significance was assessed for $\mathrm{p}<0.05$ and moderate to high levels of heterogeneity were taken into account for $\mathrm{I}^{2}>50 \%$.

\section{Results}

\section{Studies' characteristics}

Of the 26 studies identified as potentially eligible in the initial research, only 8 studies were considered eligible for quantitative synthesis, the main characteristics of the studies are summarized in Table 1 and Figure 1. Overall, this meta-analysis included data obtained from eight RCT studies that enrolled 758 patients.

Specifically, 4 RCT [14-17] compared Tele-R to patients who did not perform any rehabilitation and 3 RCT $[9,18,19]$ compared Tele-R to an active group who perform center based rehabilitation. The Vasalipoulou et al.' study [20] included both groups and data were analyzed according the group to which they belonged.

\section{Effect of tele-rehabilitation vs control group on exercise tolerance}

Four studies $[14,16,17,20]$ were included in the evaluation of the distance traveled at 6MWT after Tele-R compared to CTRL with an improvement of 33 meters (CI: $-9,+75 ; \mathrm{p}=0.123$ ), but a high level of heterogeneity $\left(\mathrm{I}^{2}=82 \%, \mathrm{p} .<0.01\right)$ (Figure $\left.2 \mathrm{a}\right)$. Removing the Cameron-Tucker et al.'s study [16] the improvement turns out to be 48 meters (CI: 24,$72 ; \mathrm{p}<0.001)$ and the heterogeneity decreased to a not significant level $\left(\mathrm{I}^{2}=49 \%, \mathrm{p}<0.35\right)$ (Figure $2 \mathrm{~b}$ ). 


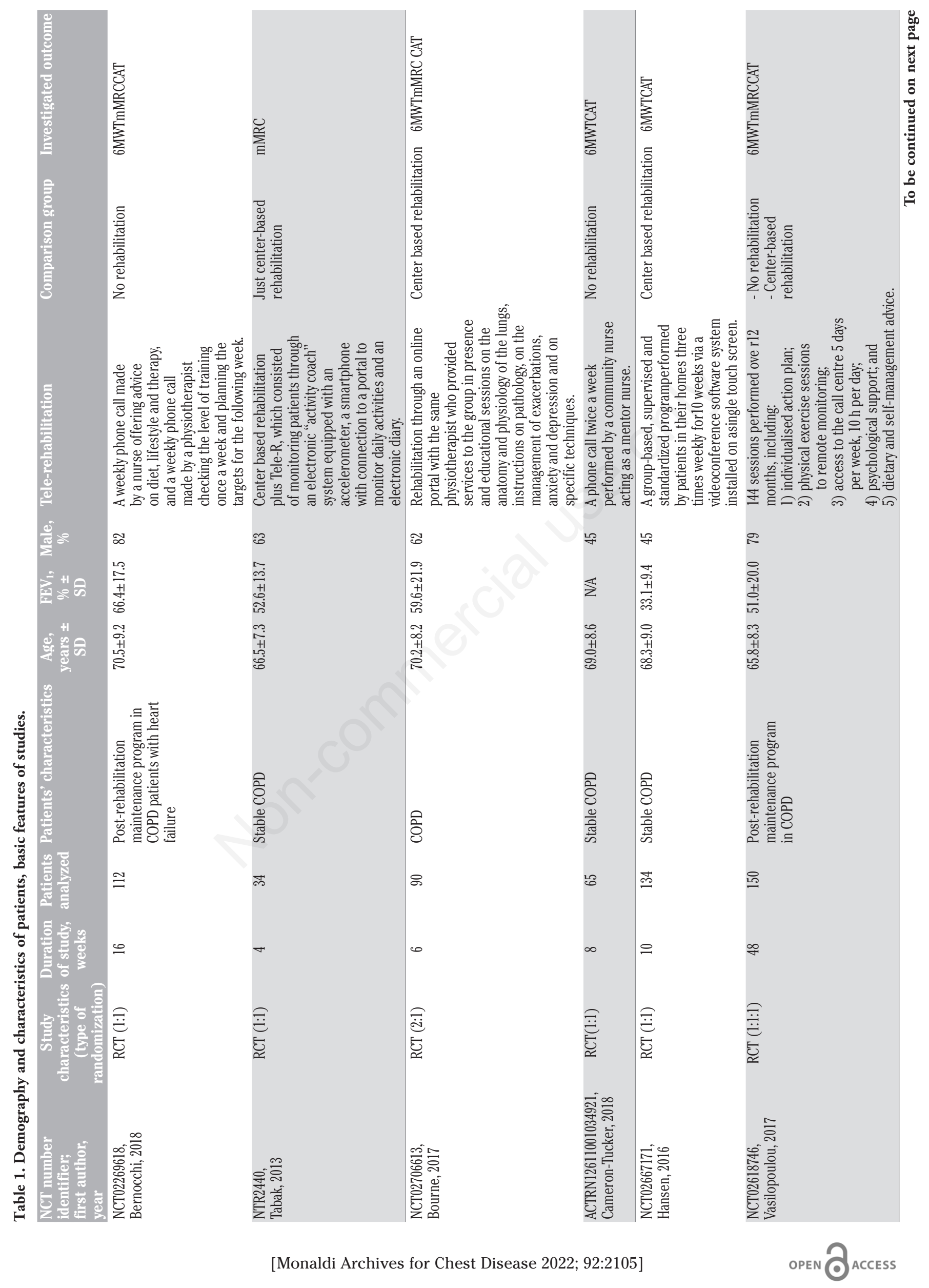




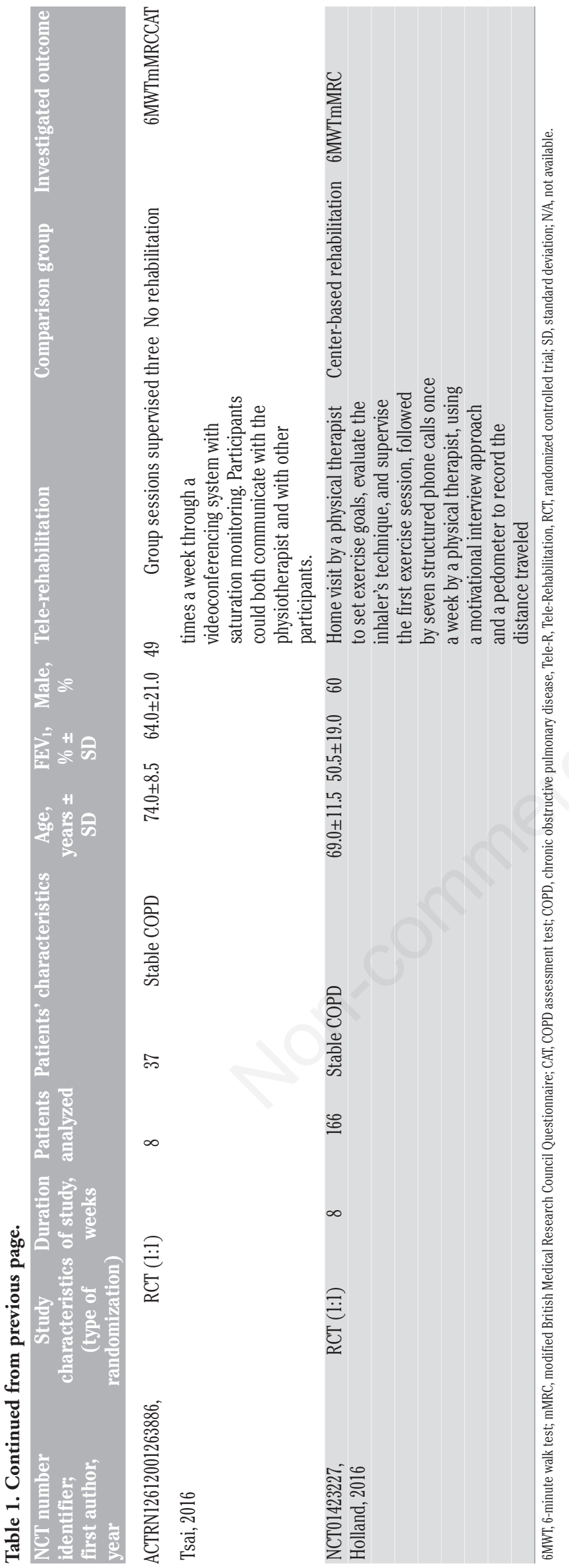

\section{Effect of tele-rehabilitation vs control group on PRO}

Four studies $[14,15,17,20]$ were included in the assessment of mMRC after Tele-R compared to CTRL with an improvement of $0.75 \mathrm{U}$ (CI: $-1.43,-0.08 ; \mathrm{p}=0.029)$, but with a high level of heterogeneity $\left(\mathrm{I}^{2}=91 \%, \mathrm{p}<0.001\right)$ (Figure $\left.3 \mathrm{a}\right)$. Removing the Bernocchi's study [14] the improvement turns out to be $-1.02 \mathrm{U}$ (CI: -1.49 , $0.59 ; \mathrm{p}<0.001)$, but the heterogeneity decreased to a moderate grade $\left(\mathrm{I}^{2}=69 \%, \mathrm{p}=0.04\right)$.

Four studies $[14,16,17,20]$ were included in the evaluation of CAT after telerehabilitation compared to CTRL with an improvement of $-4.04 \mathrm{U}$ (CI: $-7.15,-0.93 ; \mathrm{p}=0.011)$, but with a high level of heterogeneity $\left(\mathrm{I}^{2}=77 \%, \mathrm{p}<0.005\right)$ (Figure $\left.3 \mathrm{~b}\right)$. Removing the Cameron-Tucker et al.'s study [16] the improvement turns out to be $-5.74 \mathrm{U}$ (CI: $-7.42,-0.407 ; \mathrm{p}<0.001)$, and the heterogeneity decreased to a not significant level $\left(\mathrm{I}^{2}=0 \%, \mathrm{p}=0.407\right)$ (Figure $3 \mathrm{c}$ ).

\section{Effect of tele-rehabilitation vs center-based rehabilitation on exercise tolerance}

Four studies [9,18-20] were included in the assessment of distance traveled at 6MWT (6MWTD) after Tele-R compared to center based rehabilitation with an improvement of 0.061 meters (CI: $-0.15,0.27 ; p=0.563$ ) (Figure 4a).

\section{Effect of tele-rehabilitation vs center-based rehabilitation on PRO}

Three studies $[9,18,20]$ were included in the assessment of mMRC after Tele-R compared to INT with an improvement of $0.29 \mathrm{U}$ (CI: $-0.53,+-0.47 ; \mathrm{p}=0.911$ ) (Figure 4b); the same were included in the evaluation of CAT after telerehabilitation compared to CTRL with an improvement of $-1.83 \mathrm{U}$ (CI: $-3.28,-0.38$; $\mathrm{p}=0.85$ ) (Figure $4 \mathrm{c}$ ).

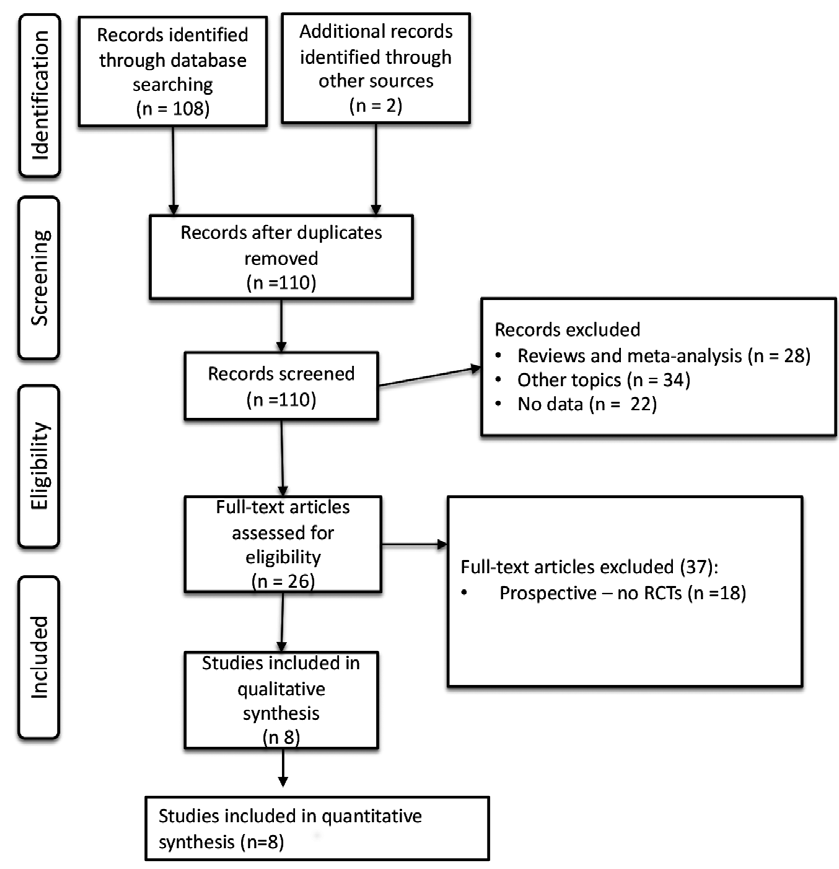

Figure 1. PRISMA-P flow diagram. 
Studies

Bernocchi 2017

Vasilopoulou 2017

Tsai 2016

Cameron - Tucker 2016

Overall $\left(\mathrm{I}^{\wedge} \mathbf{2}=\mathbf{8 2 5 7} \%, \mathrm{P}<\mathbf{0 . 0 0 1}\right)$

B)

Studies

Bernocchi 2017

Vasilopoulou 2017

Tsai 2016

Overall (I^2=486 \%, P=0.350)
Estimate (95\% C.I.)

$75.000 \quad(30.696,119.304)$

$35.400 \quad(5.332,65.468)$

$49.000(-14.794,112.794)$

$-12.000(-31.475,7.475)$

33.005

$(-8.907,74.918) \quad p .=0.123$

6 Minute Walk Test Distance, $\mathrm{m}$

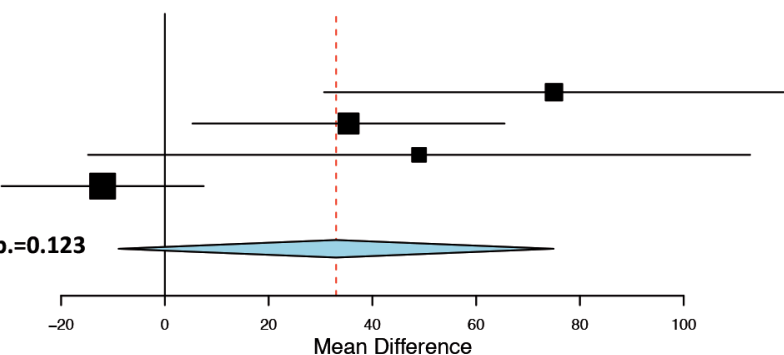

Estimate (95\% C.I.)

$75.000 \quad(30.696,119.304)$

$35.400 \quad(5.332, \quad 65.468)$

$49.000(-14.794,112.794)$

48.449

$(24.350,72.549)$ p. $<0.001$

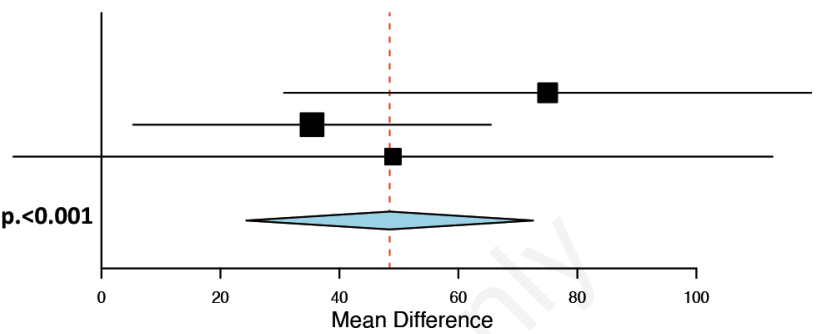

Figure 2. Effect of tele-rehabilitation $v s$ control group on exercise tolerance; a) all studies; b) without the Cameron-Tucker et al.'s study.

A) Studies

Bernocchi 2017

Tabak 2013

Vasilopoulou 2017

Tsai 2016

Overall $\left(\left.\right|^{\wedge} 2=9141 \%, P<0.001\right)$

Estimate (95\% C.I.)

$0.100(-0.192,0.392)$

$-0.650(-1.030,-0.270)$

$-1.000(-1.378,-0.622)$

$-1.600(-2.237,-0.963)$

$-0.753(-1.428,-0.078)$ p. $=0.029$
mMRC, unit

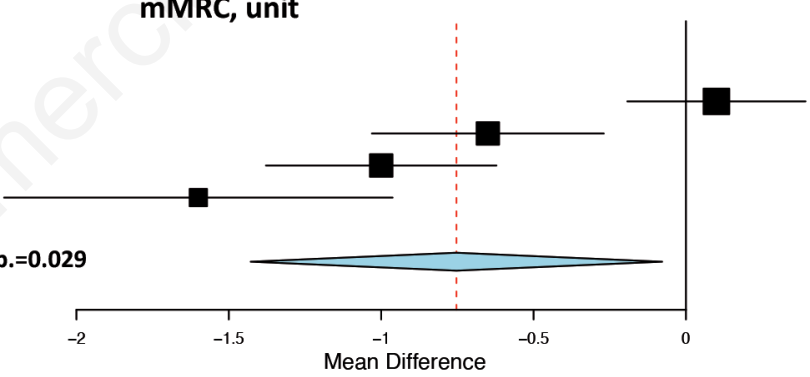

CAT, unit

B)

Studies

Estimate (95\% C.I.)

Bernocchi 2017

Vasilopoulou 2017

Tsai 2016

Cameron - Tucker 2016

$-6.900(-9.334,-4.466)$

$-5.000(-7.748,-2.252)$

$-4.000(-8.248,0.248)$

$0.000(-2.926,2.926)$

Overall (I^2=7683\%, P=0.005)

$-4.037(-7.147,-0.927) \quad p .=0.011$

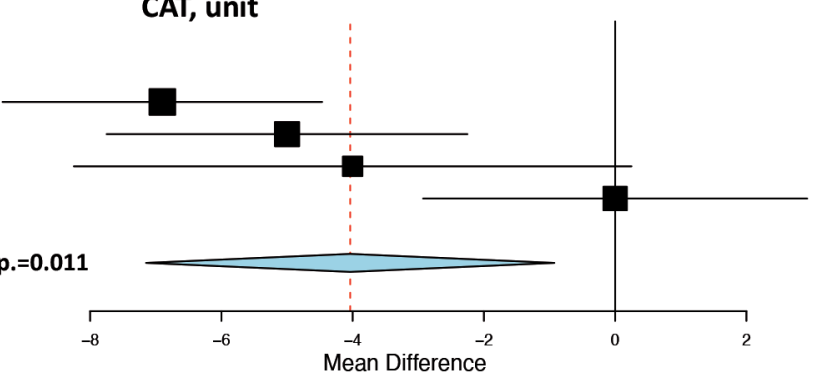

C)

Studies

Estimate (95\% C.I.)

Bernocchi 2017

Vasilopoulou 2017

Tsai 2016

$-6.900(-9.334,-4.466)$

$-5.000(-7.748,-2.252)$

$-4.000(-8.248,0.248)$

Overall (I^2=0\%,P=0.407) $-5.744(-7.418,-4.069) \quad$ p. $<0.001$

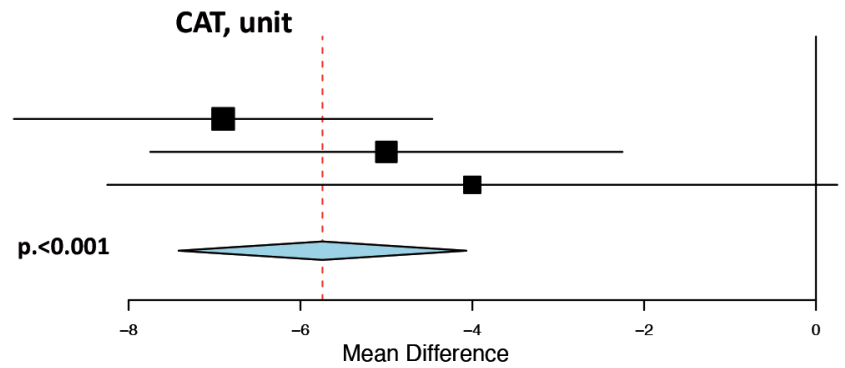

Figure 3. Effect of tele-rehabilitation $v s$ no rehabilitation group on: a) modified Medical Reserch Concil (mMRC); b) COPD assessment test (CAT); c) CAT without the Cameron-Tucker et al.'s study. 


\section{Discussion}

This study shows that Tele- $\mathrm{R}$ improves exercise tolerance and PRO in COPD patients compared to the no-rehabilitation group and that it has the same effectiveness as center-based rehabilitation performed face to face.

Tele- $\mathrm{R}$ is a new model of rehabilitation that has the advantage of being performed at home, of creating less discomfort for patients, especially fragile ones, and offers new opportunities for pulmonary rehabilitation to break down those barriers related to the patient or to geographical restrictions or mobility itself [21]. This meta-analysis shows that Tele- $\mathrm{R}$ is a valid option for patients who would not undergo any rehabilitation as it improves exercise tolerance by about 48 meters (the minimal clinical important difference (MCID) - is 25-33 meters [22]) and CAT by 5.7 points (MCID estimated around 2 points [23]) compared to no rehabilitation.

Moreover, our meta-analysis shows that Tele-R is not inferior to center-based rehabilitation and in this perspective, it could be a valid alternative for patients who cannot do rehabilitation on site or for mixed programs that provide center-based rehabilitation and Tele- $\mathrm{R}$ as maintenance therapy. However, Tele- $\mathrm{R}$ is a very generic term that includes different programs and possible methods ranging from telephone monitoring, to the application of instruments that communicate electronically to real online physiotherapy sessions [21]. Although all the studies were analyzed as a whole, they present different Tele-R programs that may justify some differences in results. For example, in the Cameron-Tucker et al.'s study [16] conducted on 65 clinically stable COPD patients, Tele-R showed no success compared to the CTRL, but Tele-R consisted of a phone call twice a week performed by a community nurse acting as a mentor and the same authors pointed out that it was not possible just with phone calls alone to monitor the effectiveness of the intervention and that probably a physiotherapist trained in this field would have been more effective. Completely opposite results were found by Tsai et al. [17], who compared Tele-R in 19 patients with stable COPD to a similar group of 17 COPD patients who did not receive physiotherapy. In this case the Tele- $\mathrm{R}$ consisted of group sessions three times a week supervised through a videoconferencing system with saturation monitoring. Participants could both communicate with the physiotherapist and with other participants. This study showed a significant improvement in exercise tolerance measured with Endurance Shuttle Walk Test, but not with 6MWT and an improvement in some PROs such as Hospital Anxiety Depression Score.

Another problem of PR is the ability to maintain long-term effects after its end, and on the other hand long-term access and utilization of PR is limited by insufficient funding, resources, or other patient-related barriers. Benefits of maintenance exercise training are evident [4], but the dropout rate is high and effects vanish after a year $[24,25]$. Tele- $R$ could be very useful as a home extension of hospital rehabilitation. Vasilopoulou et al. [20] com-
A)

Studies

Bourne 2017
Hansen 2020
Holland 2016
Vasilopoulou INT 2017
Overall (I^2=1827 \% , P=0.299)

Overall (in $=1827 \%, P=0.299)$

B)

Studies

Bourne 2017

Holland 2016

Vasilopoulou INT 2017

Overall (I^2=8472 \% , P=0.001)

c)

Studies

Bourne 2017

Hansen 2020

Vasilopoulou INT 2017

Overall $\left(I^{\wedge} 2=0 \%, P=0.850\right)$

Estimate (95\% C.I.)
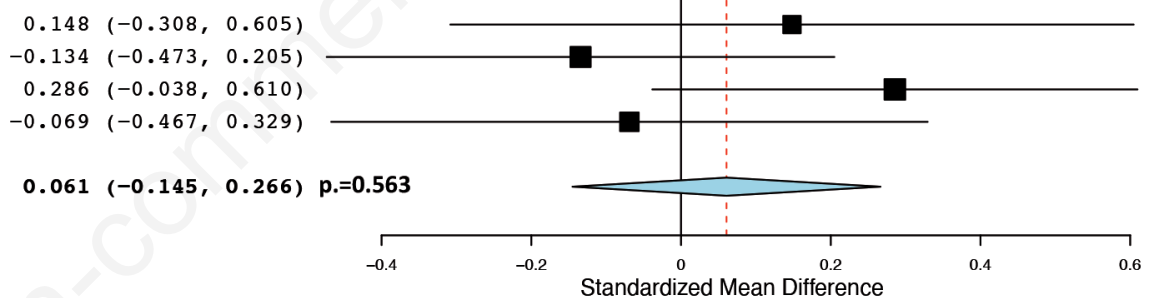

Standardized Mean Difference

mMRC, unit

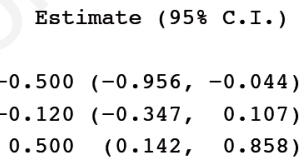

$0.500 \quad(0.142,0.858)$

$-0.029(-0.529, \quad 0.472) \quad p .=0.911$

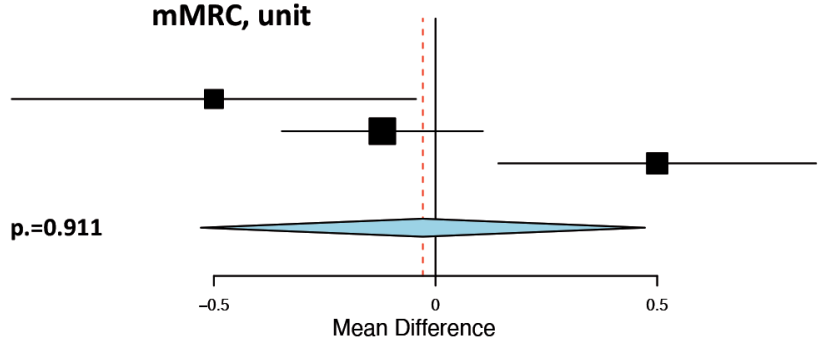

CAT, unit

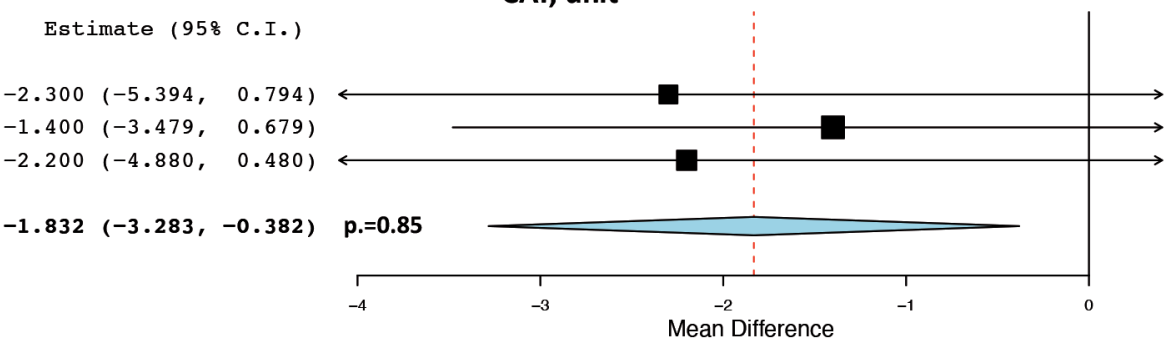

Figure 4. Effect of tele-rehabilitation $v$ s center-based rehabilitation group on: a) six minute walk test (6MWT) distance; b) modified Medical Research Council (mMRC); c) COPD assessment test (CAT). 
pared a group of 47 stable COPD patients followed for 12 months with maintenance Tele-R after 2 months of PR, with a group of patients who continued PR in the hospital (50 patients) and with another group (50 patients) who do not perform any rehabilitation, not even the initial one. The conclusions of this study are very similar to those of our meta-analysis, confirming the superiority in terms of effectiveness of Tele-R compared to the CTRL that did not perform maintenance rehabilitation, and the non-inferiority compared to center-based maintenance respiratory rehabilitation. A similar study was conducted by Bernocchi et al. [14], who explored the effect of four months of Tele-R post-rehabilitation as a maintenance program in 56 COPD patients with heart failure compared to a similar group of 56 patients followed according to normal clinical practice. This study was very positive with regard to Tele- $\mathrm{R}$ both on exercise tolerance (difference between the two groups $75 \mathrm{~m}, \mathrm{p}<0.001)$, and on different indices taken into account such as MRC $(p=0.0500)$, PASE $(p<0.005)$, Barthel $(p<0.001)$, MLHFQ $(p<0.001)$ and CAT $(p<0.001)$ and demonstrated that the Tele-R program not only had positive effects, but also that these effects were maintained for at least another two months after the suspension of the program.

Both these studies are excellent models of integration between on site rehabilitation followed by Tele-R. The availability of multiple effective models may allow patients to be offered the program in which they are most likely to succeed, which may vary based on factors such as disease stage, comorbidities, psychosocial characteristics, digital literacy, and patient choice.

Vasilopoulou et al. [20], for example, implemented patient teleconsultation based on vital sign data transmitted to an ICT webbased platform via patients' tablets and feedback to maintain progress in respect to executing home exercise drills. Differently, the Bernocchi et al.'s Tele-R consisted of a weekly phone-call by the nurse, and an exercise program monitored weekly by the physiotherapist [14]. Tabak et al. [15] monitored patients through an electronic "activity coach" system equipped with an accelerometer, a smartphone with connection to a portal to monitor daily activities and an electronic diary. Bourne et al. [18] provided Tele-R through an online portal with the same physiotherapist who provided services to the group in presence (to ensure uniformity of the service). In addition, the portal provided educational sessions on the anatomy and physiology of the lungs, lessons on pathology, on the management of exacerbations, anxiety and depression and on specific techniques such as the Active Cycle of Breathing Technique. Hansen et al. [19] supervised patients in their homes three times a week for 10 weeks via a videoconferencing software system installed on a single touch screen. Holland et al.'s [9] Tele-R consisted of a home visit by a physical therapist to set exercise goals, evaluate the inhaler technique, and supervise the first exercise session, followed by seven structured phone calls once a week by a physical therapist, using a motivational interview approach and a pedometer to record the distance traveled. Several forms of Tele-R have been experimented, from the simplest ones that consisted of just a phone call to the more complex ones equipped with accelerometers, dedicated portals, or video classes. Although new models offer the opportunity to incorporate innovations in behavioral changes and education, it is also a limit for the meta-analysis which considers any intervention as a Tele-R, without any distinction. The paucity of studies does not make any sub-analysis possible.

Another point in favor of Tele- $\mathrm{R}$ is the reduction of costs. Although this meta-analysis did not make a cost-benefit analysis, there is still limited evidence about its economic evaluation. The existing recent economic analyses from RCTs showed contrasting results in cost-effectiveness [26,27]. Haesum et al. [28] evaluated the economic impact of Tele-R in the Danish TELE- KAT project (a telehealth project on COPD patients) and demonstrated a higher cost-effectiveness of Tele-R compared to the conventional rehabilitation program, mainly reducing hospital admission, ambulatory and emergency physician contacts. However, Tele-R has a cost, which must be weighed against the economic benefits such as reduction in hospitalizations and mortality. Furthermore, if it were extended too widely, to the whole population, it could lose its economic advantages.

This meta-analysis has some limitations. Tele- $\mathrm{R}$ programs were very different in schedule and duration but, due to the scarcity of RCTs, they were considered together. As pointed out in the discussion, the term Tele- $\mathrm{R}$ includes very different interventions ranging from contacting the patient every day by phone to integrated programs including pre -program visits and education and home instrumentation. All these differences probably have an impact on the effectiveness of Tele-R but currently, due to the few studies, it is not possible to know which intervention is better, nor if there are ineffective interventions. Moreover, the duration of Tele-R programs ranges from 4 to 48 weeks. It was not possible to normalize this data because it is not possible to assume a linear growth of the parameters considered (tolerance to the exercise or dyspnea) during the rehabilitation program, therefore only the parameters at the beginning and at the end of the intervention were considered.

\section{Conclusions}

This study demonstrates the effectiveness of Tele- $\mathrm{R}$ in improving stress tolerance and symptom control in COPD patients compared to the CTRL and demonstrates the non-inferiority of Tele-R compared to respiratory rehabilitation performed in presence. The scarcity of available studies does not allow to date to distinguish the different effectiveness between the different Tele-R programs in terms of necessary equipment, exercises and duration of the program.

\section{References}

1. Spruit MA, Singh SJ, Garvey C, et al. An Official American Thoracic Society/European Respiratory Society Statement: Key Concepts and Advances in Pulmonary Rehabilitation. Am J Respir Crit Care Med 2013;188:e13-e64.

2. Lacasse Y, Martin S, Lasserson TJ, et al. Meta-analysis of respiratory rehabilitation in chronic obstructive pulmonary disease. A Cochrane systematic review. Eura Medicophys 2007;43:475-85.

3. Pitta F, Troosters T, Probst VS, et al. Are patients with COPD more active after pulmonary rehabilitation? Chest 2008; 134:273-280.

4. Rochester CL, Vogiatzis I, Holland AE, et al. An Official American Thoracic Society/European Respiratory Society Policy Statement: Enhancing Implementation, Use, and Delivery of Pulmonary Rehabilitation. Am J Respir Crit Care Med 2015;192:1373-86.

5. Siddiq MAB, Rathore FA, Clegg D, et al. Pulmonary Rehabilitation in COVID-19 patients: A scoping review of current practice and its application during the pandemic. Turk J Phys Med Rehabil 2020;66:480-94.

6. Peretti A, Amenta F, Tayebati SK, et al. Telerehabilitation: Review of the state-of-the-art and areas of application. JMIR Rehabil Assist Technol 2017;4:e7. 
7. Moher D, Liberati A, Tetzlaff J, et al. Preferred reporting items for systematic reviews and meta-analyses: the PRISMA statement. PLoS Med 2009;6:e1000097.

8. Moher D, Shamseer L, Clarke M, et al. Preferred reporting items for systematic review and meta-analysis protocols (PRISMA-P) 2015 statement. Syst Rev 2015;4:1

9. Holland AE, Mahal A, Hill CJ, et al. Home-based rehabilitation for COPD using minimal resources: a randomised, controlled equivalence trial. Thorax 2017;72:57-65.

10. Cox NS, Dal Corso S, Hansen H, et al. Telerehabilitation for chronic respiratory disease. Cochrane Database Syst Rev 2021;1:CD013040.

11. Pedder H, Sarri G, Keeney E, et al. Data extraction for complex meta-analysis (DECiMAL) guide. Syst Rev 2016;5:212.

12. Wallace BC, Dahabreh IJ, Trikalinos TA, et al. Closing the gap between methodologists and end-users: $\mathrm{R}$ as a computational back-end. J Stat Soft 2012;49:1-15.

13. Calzetta L, Rogliani P, Matera MG, et al. A Systematic review with meta-analysis of dual bronchodilation with LAMA/LABA for the treatment of stable COPD. Chest 2016;149:118-96.

14. Bernocchi P, Vitacca M, La Rovere MT, et al. Home-based telerehabilitation in older patients with chronic obstructive pulmonary disease and heart failure: a randomised controlled trial. Age Ageing 2018;47:82-8.

15. Tabak M, Vollenbroek-Hutten MM, van der Valk PD, et al. A telerehabilitation intervention for patients with Chronic Obstructive Pulmonary Disease: a randomized controlled pilot trial. Clin Rehabil 2014;28:582-91.

16. Cameron-Tucker H, Wood-Baker R, Joseph L, et al. A randomized controlled trial of telephone-mentoring with home-based walking preceding rehabilitation in COPD. COPD 2016;11:1991-2000.

17. Tsai LLY, McNamara RJ, Moddel C, et al. Home-based telerehabilitation via real-time videoconferencing improves endurance exercise capacity in patients with COPD: The randomized controlled TeleR Study: Telerehabilitation in patients with COPD. Respirology 2017;22:699-707.
18. Bourne S, DeVos R, North M, et al. Online versus face-to-face pulmonary rehabilitation for patients with chronic obstructive pulmonary disease: randomised controlled trial. BMJ Open 2017;7:e014580.

19. Hansen H, Bieler T, Beyer N, et al. Supervised pulmonary telerehabilitation versus pulmonary rehabilitation in severe COPD: a randomised multicentre trial. Thorax 2020;75:413-21.

20. Vasilopoulou M, Papaioannou AI, Kaltsakas G, et al. Homebased maintenance tele-rehabilitation reduces the risk for acute exacerbations of COPD, hospitalisations and emergency department visits. Eur Respir J 2017;49:1602129.

21. Holland AE, Cox NS, Houchen-Wolloff L, et al. Defining modern pulmonary rehabilitation. An Official American Thoracic Society Workshop Report. Ann Am Thorac Soc 2021;18:e12-e29.

22. Puente-Maestu L, Palange P, Casaburi R, et al. Use of exercise testing in the evaluation of interventional efficacy: an official ERS statement. Eur Respir J 2016;47:429-60.

23. Dodd JW, Hogg L, Nolan J, et al. The COPD assessment test (CAT): response to pulmonary rehabilitation. A multicentre, prospective study. Thorax 2011;66:425-9.

24. Alison JA, McKeough ZJ, Johnston K, et al. Australian and New Zealand pulmonary rehabilitation guidelines. Respirology 2017;22:800-19.

25. Güell M-R, Cejudo P, Ortega F, et al. Benefits of long-term pulmonary rehabilitation maintenance program in patients with severe chronic obstructive pulmonary disease. Three-year follow-up. Am J Respir Crit Care Med 2017;195:622-9.

26. Barbosa MT, Sousa CS, Morais-Almeida M, et al. Telemedicine in COPD: An overview by topics. COPD 2020;17:601-17.

27. Longacre CF, Nyman JA, Visscher SL, et al. Cost-effectiveness of the Collaborative Care to Preserve Performance in Cancer (COPE) trial tele-rehabilitation interventions for patients with advanced cancers. Cancer Med 2020;9:2723-31.

28. Haesum LKE, Soerensen N, Dinesen B, et al. Cost-utility analysis of a telerehabilitation program: A case study of COPD patients. Telemed J E Health 2012;18:688-92. 